\title{
Transformation and Upgrading of Social Support System for the Elderly Health Based on Dissipative Structure Theory
}

\author{
Dongmei Liang ${ }^{1, *}$
}

\author{
${ }^{1}$ School of Public Affairs and Administration, University of Electronic Science and Technology of China, Chengdu, \\ Sichuan 611713, China \\ *Corresponding author. Email:1dm1210@std.uestc.edu.cn
}

\begin{abstract}
China has ushered in aging society, even advanced aging society, given its rapid expansion of elder population and world-leading aging rate. As the elderly population ages, the number of elderly people suffering from disability, semi-disability and chronic diseases is also mounting, exacerbating the health problems of the elderly. In order to relieve the pressure of the traditional family, the theory of dissipative structure is employed in this paper to study the social support system for elderly health. How to realize win-win cooperation among government, community, family and individual under the aging background is also elaborated to improve the function and structure of the system.
\end{abstract}

Keywords: aging, dissipative structure, social support system for elderly health

\section{INTRODUCTION}

The century has witnessed China's economic and social development, better social security system including pension security and medical security, changing population structure, more elders, and increasingly severe aging issue. According to National Bureau of Statistics in China, its total population exceeded 1.4 billion as of 2019, with 253.88 million people aged 60 and above, accounting for $18.1 \%$. Compared with the end of 2018, the population aged 60 and above increased by 4.39 million, or 0.25 percentage points. The aging of the population will lead to inadequate labor supply and huge pension burden. Meanwhile, the challenges abounds, such as the declining health status, lack of financial resources and narrow social scope of the elderly.

Social support system for elderly health is a complex system involving multiple subjects such as family, community, government and enterprise. Special rules apply to its development and evolution, and more attention are needed. In response to the aging crisis, the report to the 19th National Congress of the Communist Party of China (CPC) proposed to "build a policy system and social environment for supporting the elderly, showing filial piety and respect for the elderly, promote the combination of medical care and nursing care, and accelerate the development of undertakings and industries for the elderly". At the fourth Plenary Session of the 19th CPC Central Committee, it was proposed to adhere to and improve the social governance system featuring co-construction, cogovernance and shared benefits. As a result, all regions encourage social participation into old-age care in the spirit of co-construction, co-governance and shared benefits, and incorporate the cultivation of social atmosphere of showing respect and filial piety for the elderly and the establishment of care and service system for the elderly into government assessment system. Despite the progress made in elderly health thanks to public attention, the lack of autonomy and vitality is highlighted given its top-down nature. Therefore, this paper aims to provide new theoretical insight for the research on social support system for elderly health based on dissipative structure theory.

\section{THEORETICAL BASIS AND LITERATURE REVIEW}

\section{A. Previous studies on the elderly}

More attention has been paid to the elderly among scholars in recent years. The latter have studied the improvement of public policies for the elderly, pension service system and pension model from a macro perspective, as well as factors affecting their health and life satisfaction from a micro perspective.

In terms of the public policy for the aged, $\mathrm{Hu}$ Xiaowu [1], Han Ye [2], Li Yongping [3] and other scholars predicted various future issues encountered by cities in China's, and proposed "pluralistic integration" model of collective-led elderly care in rural communities. Yu Xiao [4] and Jia Yujiao [5] paid more 
attention to the smart pension model. At the micro level, Xiang Yunhua and Hu Tiantian empirically tested the differences in medical consumption behavior of the elderly in China's rural areas and main influencing factors from the perspective of income inequality and basic medical insurance by employing the data of China Family Panel Studies in 2018 [6].

As for social support for the elderly, Fang Hao [7], Liu Hao [8], Zhou Wei [9], Wang Fubaihui [10] and other scholars conducted empirical analysis on the role of subjective and objective support on the health status of the elderly, such as children support, social capital, and friend support. Qu Xiaomin took mental health and physical health as mediating variables and expanded the research mechanism of social support on life satisfaction of the elderly [11].

Social support system is a system consisting of subject, object, social support tool and social support content. It is formed by the comprehensive effect of individual, family, society and government factors, which can meet the diversified material and spiritual needs of the elderly, and has the functions of selfdevelopment and self-repair. Zhao Lixin [12], Zhang Banghui [13] et al. provided reference for the construction of complete social support network for the elderly through questionnaire survey. Peng Qingyun and Zhao Xianghong analyzed the reasons for the frequent loss of the elderly through the social support system, and believed that the family, community and news network media should be rationally utilized to construct social support system to prevent such loss.

Therefore, the application of social support system to the study of the elderly is of practical significance to realize the social governance featuring "coconstruction, co-governance and shared benefits". However, social support content is taken as research object, and few scholars have studied the formation rules and existing problems of the social support system itself. Therefore, this paper introduces the theory of dissipative structure to sort out the public policies for the elderly since the founding of new China, and finds the evolution rules and characteristics of the social support system, so as to provide theoretical basis for improving the system.

\section{B. Dissipative structure theory}

Dissipative structure is a system evolution process from disorder to order. When an open system is out of balance, and the system constantly exchange energy and material with external environment, under whose stimulus the factors within the system will fluctuate due to reaching a certain threshold, and the system will be different. As a result, the system will transform from the original chaotic state to a new ordered state in terms of time, space and function. [15]
The formation of dissipative structure must meet the following four conditions:

- Open system. When the self-organized dissipative structure is spontaneously formed in the system, an open system must be guaranteed to exchange inside and outside the system, which provides the possibility for entropy decrease.

- The equilibrium state should be avoided to maintain the continuity of self-organizing behavior.

- Internal fluctuation can be used to activate the internal system development.

- The nonlinear interaction is guaranteed, which amplifies the fluctuation movement within the system, promotes the interaction and coupling of multiple factors within the organizational system, and finally forms the dissipative structure.

The core of the dissipation mechanism is the negative entropy flow generated in the exchange process. The change of system entropy includes two processes: one is the entropy exchange (DES) in the process of internal and external exchange, and the other is the change of entropy that is always positive inside the system, which is called entropy generation (DIS). Therefore, the formula of the algebraic sum of system entropy change is: $d s=$ des + dis. In this process, the negative entropy generated by the internal and external exchange of the system will greatly affect the system, that is, the negative entropy is generated in the process. At this time, the change of the entropy decreases and gradually changes from positive to negative. New trajectories are generated in the nonlinear fluctuation changes of the system, and an ordered state is finally formed. As the internal and external exchange continues, a "negative entropy flow" will continue to form, and finally a stable dissipative structure will be formed in the system. [16]

The health social support system for the elderly satisfies these four conditions of dissipative structure. Under the open system of health social support, the external environment stimulates the internal factors and continuously competes with the internal factors. As a parameter is introduced, the negative entropy associated with multiple exchanges of information, energy, and matter increases to such an extent that the internal entropy cancels out and the system fluctuates. The system under the nonlinear effect changes greatly, and then this parameter will lead the system to change the direction of development and evolution. 


\section{EVOLUTION OF THE HEALTH SOCIAL SUPPORT SYSTEM FOR THE ELDERLY}

The development of the health social support system for the elderly mainly includes the structural transformation of time, structure and function. The support system is irreversible as for time, so this paper focuses on the evolution of the system in terms of structure and function.

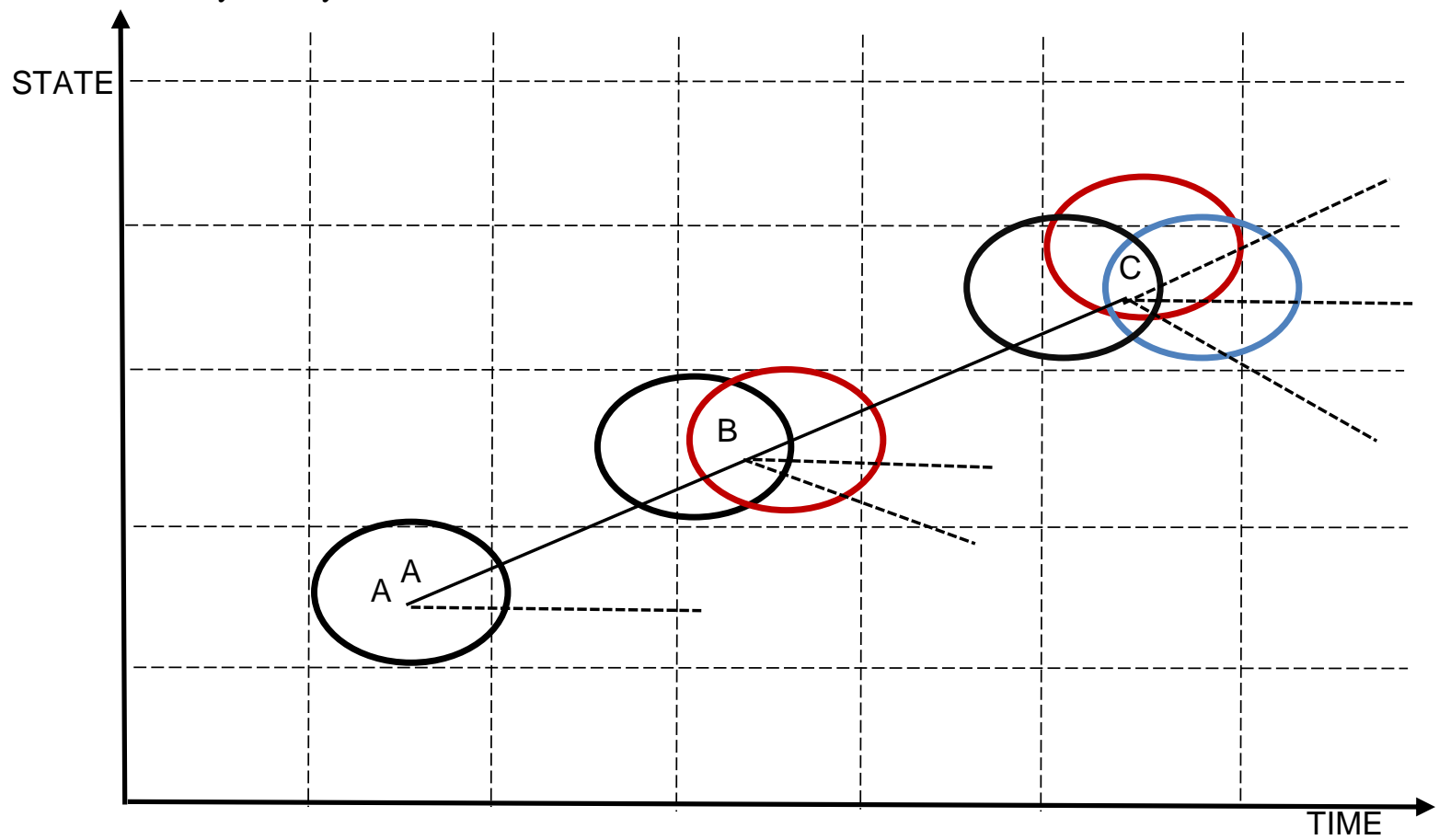

Fig. 1. Changes in the spatial and functional structure of social support systems.

In terms of spatial and functional structure, social support system for elderly health undergoes three development stages, namely, A, B and C in "Fig. 1". The social support subjects and characteristics of the social support system vary with different stages, so does functions.

\section{A. Characteristics and functional structure of social support system for elderly health in the initial stage}

The initial phase (A) is the first phase of social support development. Online materials and government policy texts prove that before 1996, China's public policies at the national macro level seldom mentioned the health of the elderly, the rights and interests of the elderly, and the environment of filial piety and respect for the elderly, etc. Therefore, this paper takes the period from 1949 to 1996 as stage A. The stage is characterized by smallness and weakness, that is, the scope of social activities is small and the strength of social support is weak, which is mainly composed of family support. Kinship and geographical relation are heavily relied in this stage, where households offered the elderly with tangible support and emotional support, namely alimony and household care by children or relatives. The exchange of information in a small scope is also realized, including family and neighborhood, and the exchange of information is slow.

At this stage, the government's policy support is weak. Before 1996, when the Ministry of Labor issued the "Protection of the Rights and Interests of the Elderly of the People's Republic of China", China lacked constitutional and legal policy support for the legitimate rights and interests of the elderly, not to mention policy support for medical care and poverty for the elderly. As for the organizational mode, the communication scope spontaneously formed by the family, namely the unitary network pattern with the elderly family as the core, including neighbors, etc., which cripples the social support system for elderly health, leads to health inequality in the context of uneven family resources, and constrains the improvement of the elderly mental health and happiness.

\section{B. Characteristics and functional structure of social} support system for elderly health in the second stage

The second stage (B) refers to the period from 1996 to the present, which is referred to in this paper as the medium-level support stage. The region where B is located is covered by family support and government 
support, in which the government takes the lead and the family is the most important social support subject. China is at this stage.

In this stage, the "9073" old-age care pattern was formed. Policy tools were used as the external stimulus source to gradually reduce the proportion of families in social support, and social forces were continuously promoted to intervene, so as to promote the evolution of social support system from stage B to stage C. In 1996, the Ministry of Labor issued Protection of the Rights and Interests of the Elderly of the People's Republic of China. China National Committee on Aging and the Ministry of Civil Affairs have also launched policy documents such as the Opinions on Strengthening Preferential Treatment for Elderly and the Opinions on Supporting Social Forces in Setting up Social Welfare Institutions. The social security system, such as medical insurance for the elderly and medical assistance for the poor, has been improved, and a large number of neglected social forces have entered the social support system for the elderly. The increasingly severe aging of population has prompted the government to pay more attention to elderly health. After 2011, the State Council issued the Plan for the Construction of Social Old-age Service System (2011-2015) and the Plan for the Construction of Community Service System (20112015), which put forward integration of medical treatment and elderly support, and launched the longterm care insurance system in 2015. In his report to the 19th National Congress of the Communist Party of China (CPC), General Secretary Xi Jinping even proposed the concept of "co-construction, cogovernance and shared benefits", elevating social forces in a crucial position. It's proved that at this stage, the policy not only changed the organization mode of family, government and society in the social support system, but also changed the concept of social endowment, continuously promoting the integration of health and endowment resources.

\section{Characteristics and functional structure of social support system for elderly health in the third stage}

The third stage (C) is the strong support phase, which is an ideal area fully covered by family support, government support and social support, and is a phase that benefits from the stimulus of government policy tools. The main feature of this stage is to get rid of the government control and the formation of social governance of "co-construction, sharing and cogovernance". The social responsibility of social organizations is enhanced, and they participated in social support system for the elderly health. The introduction of new social forces makes the system produce negative entropy, and the healthy social support system fluctuates under the action of "negative entropy flow". At the same time, government policy tools can be employed to upgrade and transform the existing system, and to create social atmosphere of respecting and loving the elderly, so as to make internal and external factors of the health social support system compete with each other, and finally shorten the evolution time of the system. It is necessary to reflect on the organization mode, science and technology of the health social support system, so as to avoid the buck-passing or vicious competition among families, society and government in the system. Otherwise, the strong social support system in stage $\mathrm{C}$ may evolve to a low level, and eventually the system will lose order, return to disorder, and finally perish.

\section{SUGGESTIONS ON IMPROVING THE SOCIAL SUPPORT SYSTEM FOR ELDERLY HEALTH}

Understanding the evolution of the health social support system for the elderly and the existing problems is helpful to improve the system.

\section{A. Building a network governance model for the health of the elderly}

The organizational mode should be innovated, the structure of the three supporting bodies should be adjusted reasonably, and the network governance model of shared health for the elderly should be built based on the pattern of 9073. The functions and roles of government, family and society in the social support system should be clarified, and other social organizations should be studied in depth. On this basis, this paper argues that in the stage of middle-level social support, the supporting role of the community associated with the family must be considered, and the community should be used as the carrier to embed the market mechanism of elderly health, so as to build a healthy ecosystem for the elderly in which all kinds of organizations and resources are accompanied and interdependent. Through competition mechanism, an elderly health system with market operation is embedded in the community to form a network and three-dimensional service mode with community as support and multi-dimensional resources gathering.

Under the three-dimensional service mode supported by the community, the functions of the government, community management institutions (neighborhood committee, neighborhood committee, owners committee, residents autonomous organizations and intermediary organizations, etc.), enterprises, families and others should be clarified to create a healthy and elderly credit system.

\section{B. Moving forward the health threshold for the elderly}

In terms of policy innovation, the lack of targeted health policies for the elderly should be made up, and the health threshold for the elderly should be moved forward to focus on prevention. 
The first is to increase the penetration of medical insurance. On the basis of ensuring basic medical care and strengthening medical treatment for serious diseases, the construction of long-term care insurance system should be promoted. The mental health and life happiness of the elderly can be adopted as indicators for the health of the elderly, and the in-depth integration of medical and old-age resources could be promoted. Secondly, the innovative fiscal and tax system is used as a lever to move social capital into the field of elderly health, breaking the original balance between family support and government support. Finally, health policy is skewed towards families and communities. Preferential long-term family pension policies have been formed to match children, such as the one-child holiday. In addition, a safe and efficient community support mechanism for the elderly will be built with the help of the policy. The residents' committee will take the lead in providing various services such as accommodation and nursing care for the elderly with the carrier of nursing homes and community day care centers, and the community will be encouraged to take care of the elderly and reduce the burden of children.

\section{Building technology complementarity mechanisms}

In terms of technological innovation, peopleoriented concept should be adhered to, and technology complementary mechanism should be established. In view of the existing neglect of aging technology, a benign interactive system of "government-led, enterprise-joined, market-operated and societyparticipated" must be established. The government should provide financial and policy support for the reform of elderly-oriented technology. On the one hand, financial and patent tax incentives should be provided to enterprises. On the other hand, financial support should be given to investigate the technology demand of the elderly, establish the incubation area of elderlyoriented technology innovation, and promote the supply of products suitable for aging.

Enterprises should establish brand awareness, and consumers' temptation in the market can be used to adjust the research and development direction of healthy and intelligent products for the elderly, and "intelligent" can be applied to all aspects of the life of the elderly. As a participant in the technological innovation and upgrading of healthy aging technology for the elderly, the society should cooperate with the technological innovation plan of the government within a reasonable scope, support the social investigation of the technological demand for the elderly by enterprises and universities, and treat the development of existing aging technology with an open, healthy and positive attitude.

\section{Establishing a social service platform to help the elderly engage in work}

The concept of the combination of health self-help and other assistance for the elderly is followed. With the elderly as the core and the Internet as the means, on the basis of guaranteeing the physiological health of the elderly, attention is paid to group life, the scope of social activities of the elderly is expanded, and social service for the elderly is established. On the one hand, the work demands of the elderly should be respected. Taking the will of the elderly as an indicator, a work information platform for the elderly should be established and improved. With a balance between "demand" and "supply" as the orientation, customized and personalized social work opportunities should be provided for the elderly, and the elderly should be highly recognized for their work. On the other hand, the resources of the social support system should be reasonably allocated, with the government, the community and social organizations as the main subjects, and the responsibilities and obligations of the three parties should be defined. The government can provide the elderly with work opportunities matching their age, gender, education background and other individual conditions through outsourcing, while the society design social work with reasonable salary and labor such as elder volunteer activities and community services for the elderly through community neighborhood committees, property management and other relevant departments.

\section{CONCLUSION}

China's social support system for elderly health is at a moderate level, which is mainly stimulated by government policy tools. Its development depends on whether external factors can enter the system smoothly, collide with the system and produce fluctuations. The upgrade of the system requires the stimulation of policy tools, innovation of pension concept, change of organizational mode, and enhancement of technology utilization, so as to generate "negative entropy flow" in the dissipative structure and gradually realize order through disordered change.

\section{References}

[1] Hu Xiaowu. The conceptional changes in urban policies for the elderly in the context of aging society [J]. Urban Problems, 2008, 000(007):67-72. (in Chinese)

[2] Han Ye, Fu Jiaping. China's Old-age Service Policy Supply: Evolution, Governance Framework, Future Direction [J] Lanzhou Academic Journal, 2020(9). (in Chinese)

[3] Li Yongping. "Pluralistic Integration": A Model of CollectiveLed Elderly Care in Rural Communities - A Case Study of Rural Geracomium in Southern Fujian [J]. Truth Seeking, 2020(05): 96-108+112. (in Chinese) 
[4] Yu Xiao, Sun Yue. The Model of "Internet + Old-age Service": The Innovative Development of Old-age Service in New Era [J]. Population Journal. 2017(01). (in Chinese)

[5] Jia Yujiao, Wang Cong. The Construction of Demand-oriented Smart Home Care Service System [J]. Inner Mongolia Social Sciences, 2020, 41(05): 166-172+213. (in Chinese)

[6] Xiang Yunhua, Hu Tiantian. Rural elderly medical consumption behavior under the "Healthy China" strategy: from the perspective of income inequality and basic medical insurance [J]. Journal of Central China Normal University (Humanities and Social Science), 2020 (5). (in Chinese)

[7] Fang Hao. An Empirical Study of the Influence of Social Capital on the Health of Urban and Rural Elderly - Based on CGSS Mixed Section Data [J]. Journal of Huazhong Agricultural University(Social Sciences Edition), 2020(02): 8897+166. (in Chinese)

[8] Liu Hao, Li Qiang. Impact of Children's Taking Care of the Mental Health of the Rural Disabled Elderly: Micro-Evidence from Chinese Families [J]. Journal of Yunnan Minzu University(Social Sciences), 2020, 37(02): 104-116. (in Chinese)

[9] Zhou Wei, Hong Zijing, Hu Rongrong, Zhu Tingting, Liu Shen, Zhang Lin. Parent-child Support and Elders' Depression: Roles of Sense of Security and Emotional Expression [J]. Psychological Development and Education, 2020, 36(02) :249256. (in Chinese)

[10] Wang Fubaihui, Huang Yingjiao, Li Yaqian, Pan Yue, Yang Fan. Friend Network Support and Exercise Participation of the Elderly: The Mediating Role of Social Adaptation [J]. China Sport Science and Technology, 2020,56(09):75-81. (in Chinese)

[11] Qu Xiaomin. The Influence Mechanism of Social Support on Life Satisfaction of the Elderly -- Introduce Physical Health and Mental Health as Mediation [J]. Population Journal, 2016, 038(002): 49-60. (in Chinese)

[12] Zhao Lixin. Study on Social Support System of Community Service -oriented Home Caring of the Aged [J]. Population Journal, 2009(6):41-46. (in Chinese)

[13] Zhang Banghui, Li Wei. Construction of social support system based on psychological needs of left-behind elderly in rural areas [J]. Journal of Chongqing University (Social Sciences Edition), 2018, 24(01): 145-154. (in Chinese)

[14] Peng Qingyun, Zhao Xianghong, Wei Sijia. The Elderly Getting Lost and the Construction of Its Social Support System [J]. Academic Journal of Zhongzhou, 2019(12): 89-93. (in Chinese)

[15] Shen Xiaofeng, Hu Gang, Jiang Lu. Establishment of theory of dissipative structure [J]. Studies in Dialectics of Nature, 1986(6):45-49. (in Chinese)

[16] Edited by Shen Wei. Dissipative structure, self-organization, catastrophe theory and earth science [M] Beijing: Geological Publishing House, 2008: 3. (in Chinese) 